PROCEEDINGS OF THE

AMERICAN MATHEMATICAL SOCIETY

Volume 135, Number 6, June 2007, Pages 1803-1815

S 0002-9939(06)08670-9

Article electronically published on December 28, 2006

\title{
SOLUTIONS GLOBALES DE CERTAINES ÉQUATIONS DE FUCHS NON LINÉAIRES DANS LES ESPACES DE GEVREY
}

FAIZA DERRAB

(Communicated by David S. Tartakoff)

\begin{abstract}
We consider nonlinear partial differential equations with several Fuchsian variables of type $a\left(t, D_{t}\right) u(t, x)=f(t, x, D u(t, x))$, where $a\left(t, D_{t}\right)$ is a Fuchsian principal part of weight zero. We prove existence and uniqueness of a global solution to this problem in the space of holomorphic functions with respect to the Fuchsian variable $t$ and in Gevrey spaces with respect to the other variable $x$. The method of proof is based on the application of the fixed point theorem in some Banach algebras defined by majorant functions that are suitable to this kind of equation.
\end{abstract}

\section{INTRODUCTION}

Le problème de l'existence et de l'unicité d'une solution holomorphe locale pour des équations de Fuchs aux dérivées partielles non linéaires, a été étudié dans les travaux de R. Gérard et H. Tahara [5], N. S. Madi et M. Yoshino 9] et M. Miyake [10, mais leurs méthodes de démonstration utilisent des techniques trés calculatoires.

P. Pongérard [11] a simplifié la preuve de ces résultats, donnés dans des espaces de fonctions holomorphes, à celle du théorème du point fixe qui repose sur le formalisme des fonctions majorantes développé par $\mathrm{C}$. Wagschal [13 pour la résolution locale du problème de Goursat dans les espaces de fonctions holomorphes et de Gevrey. P. Pongérard a considéré un opérateur de Fuchs non linéaire d'un type plus général que ceux traités dans les travaux précédents, puis, en se basant sur l'idée de M. Gevrey [6] qui consiste à déduire les proriétés des espaces de Gevrey de celles des espaces de fonctions analytiques, l'auteur a déduit de la première partie de son travail, l'existence et l'unicité d'une solution locale dans les espaces de Gevrey classiques introduits dans 13 .

Dans ce travail, on montre l'existence et l'unicité globales de la solution d'une équation aux dérivées partielles non linéaire de type de Fuchs. On établit cette solution dans des espaces de fonctions holomorphes par rapport à la variable Fuchsienne et de Gevrey par rapport aux autres variables, cette résolution n'apparait pas dans les travaux précédents. Notre démarche consiste à utiliser les techniques de [13] qui sont basées sur l'application du théorème du point fixe dans des algèbres de Banach, qu'on définit par des fonctions majorantes convenables pour l'étude de

Received by the editors March 4, 2005 and, in revised form, February 6, 2006.

2000 Mathematics Subject Classification. Primary 35A05; Secondary 35G20, 35A20.

Key words and phrases. Nonlinear Fuchsian partial differential equation, several Fuchsian variables, global solution, Gevrey classes, method of majorants, fixed-point theorem.

(c)2006 American Mathematical Society

Reverts to public domain 28 years from publication 
ce type d'équations et, dans lesquelles la résolution de notre problème sera réduite à la recherche des points fixes d'une certaine application qu'on construit à partir du problème initial et, qu'on montre strictement contractante. Ces fonctions majorantes seront obtenues à partir de celle introduite dans [11] à laquelle on applique la même transformée utilisée dans $[3$. Afin que notre problème soit bien posé, nous avons opté aux espaces de Gevrey $G^{(d)}$, d'indice $d \geq 1$, introduits par H. Komatsu [7] et on a considéré un opérateur Fuchsien dont la partie principale est à coefficients constants.

La méthode du point fixe a aussi été utilisée avec succès dans la résolution du problème de Cauchy linéaire asocié à des équations aux dérivées partielles Fuchsiennes dans le sens de M. S. Baouendi et C. Goulaouic [1. On cite d'abord la résolution globale de ce type de problèmes dans des espaces de fonctions entières (éventuellement d'ordre fini) par P. Pongérard dans [12]. Dans [3], les auteurs ont encore simplifié la méthode du point fixe proposée dans [12] pour la résolution globale du même problème dans la classe de fonctions holomorphes et, ont généralisé le résultat de [12] à un opérateur différentiel à plusieurs variables Fuchsiennes. Ensuite, le résultat de [12] a été généralisé dans [2] à une résolution globale dans la classe de fonctions holomorphes par rapport à la variable Fuchsienne et de classe de Gevrey $G^{\{d\}}$ (respectivement de Gevrey $G^{(d)}$ au sens de H. Komatsu 7]), d'indice $d \geq 1$, par rapport aux autres variables.

On note que les résultats d'existence globale pour des problèmes non linéaires sont rares. Dans un sens, le résultat de ce travail est une extension de celui de [11] à une version globale dans les espaces de Gevrey $G^{(d)}$ et, est une généralisation du résultat global de [2] à l'étude d'un problème non linéaire toujours dans les espaces de Gevrey $G^{(d)}$.

\section{DÉfinitions et RÉSUltat}

Soient $n$ et $q$ deux entiers naturels non nuls. On étudie des équations aux dérivées partielles non linéaires de type de Fuchs dans l'espace $\mathbb{C}^{q} \times \mathbb{R}^{n}$. Les points génériques de $\mathbb{C}^{q}$ et $\mathbb{R}^{n}$ seront notés respectivement $t=\left(t_{1}, \ldots, t_{q}\right)$ et $x=\left(x_{1}, \ldots, x_{n}\right)$.

Pour un multi-indice $\gamma=\left(\gamma_{1}, \ldots, \gamma_{q}\right) \in \mathbb{N}^{q}$, on notera $\gamma !=\left(\gamma_{1}\right) ! \ldots\left(\gamma_{q}\right)$ ! et on appellera longueur de $\gamma$ l'entier $|\gamma|=\gamma_{1}+\ldots+\gamma_{q}$. Pour deux multi-indices $\gamma$, $\beta \in \mathbb{N}^{q}$, on écrit $\gamma \leq \beta$ si $\gamma_{i} \leq \beta_{i}$ pour tout $i \in\{1, \ldots, q\}$ et $\gamma<\beta$ si $(\gamma \leq \beta$ et $\gamma \neq \beta)$.

Pour $t=\left(t_{1}, \ldots, t_{q}\right) \in \mathbb{C}^{q}$, on notera $t^{\gamma}=t_{1}^{\gamma_{1}} \ldots t_{q}^{\gamma_{q}}$ et $D_{t}^{\gamma}=D_{t_{1}}^{\gamma_{1}} \ldots D_{t_{q}}^{\gamma_{q}}$ où $D_{t_{i}}$ est l'opérateur de dérivation par rapport à la variable $t_{i}$ et on pose $t D_{t}=$ $\left(t_{1} D_{t_{1}}, \ldots, t_{q} D_{t_{q}}\right)$. De même, pour un multi-indice $\alpha=\left(\alpha_{1}, \ldots, \alpha_{n}\right) \in \mathbb{N}^{n}$ et pour $x=\left(x_{1}, \ldots, x_{n}\right) \in \mathbb{R}^{n}$, on notera $D_{x}^{\alpha}=D_{x_{1}}^{\alpha_{1}} \ldots D_{x_{n}}^{\alpha_{n}}$ où $D_{x_{j}}$ est la dérivée par rapport à la variable $x_{j}$.

Étant donné un ouvert $U$ de $\mathbb{C}^{q}$ et un ouvert $\Omega$ de $\mathbb{R}^{n}$, on note $\mathcal{C}^{\omega, \infty}(U \times \Omega)$ l'algèbre des fonctions $u: U \times \Omega \longrightarrow \mathbb{C}$ telles que:

i) pour tout $t \in U$, l'application $x \longmapsto u(t, x)$ est $\mathcal{C}^{\infty}$ dans $\Omega$,

ii) pour tout $\alpha \in \mathbb{N}^{n}$, l'application $D_{x}^{\alpha} u: U \times \Omega \longrightarrow \mathbb{C}$ est continue,

iii) pour tout $x \in \Omega$ et tout $\alpha \in \mathbb{N}^{n}$, l'application $t \longmapsto D_{x}^{\alpha} u(t, x)$ est holomorphe dans $U$.

Cette algèbre est stable par dérivation et les opérateurs $D_{t}^{\gamma}$ et $D_{x}^{\alpha}$ commutent. 
Définition 2.1. Étant donné un réel $d \geq 1$, on note $G^{\omega,(d)}(U \times \Omega)$ l'ensemble des fonctions $u \in \mathcal{C}^{\omega, \infty}(U \times \Omega)$ telles que pour tout compact $\mathcal{K} \subset U \times \Omega$, pour tout $h>0$, il existe une constante $c \geq 0$ telle que

$$
\forall \alpha \in \mathbb{N}^{n}, \max _{(t, x) \in \mathcal{K}}\left|D_{x}^{\alpha} u(t, x)\right| \leq c h^{|\alpha|}(\alpha !)^{d} .
$$

$G^{\omega,(d)}(U \times \Omega)$ est une sous-algèbre de $\mathcal{C}^{\omega, \infty}(U \times \Omega)$, stable par dérivation.

L'algèbre des fonctions $u \in G^{\omega,(d)}(U \times \Omega)$ indépendante de $t$ est notée $G^{(d)}(\Omega)$ ), il s'agit de l'espace des fonctions de classe de Gevrey au sens de H. Komatsu [7].

Étant donné $m=\left(m_{1}, \ldots, m_{q}\right) \in \mathbb{N}^{q}$, on considère un opérateur différentiel non linéaire de type de Fuchs dont la partie principale est de la forme $a\left(t, D_{t}\right)=$ $\sum_{\gamma \leq m} a_{\gamma} t^{\gamma} D_{t}^{\gamma}$, où $a_{\gamma}$, pour $\gamma \leq m$, sont des nombres complexes avec $a_{m} \neq 0$. $a\left(t, D_{t}\right)$ est donc une partie principale Fuchsienne d'ordre $m$ et de poids zéro.

On associe à l'opérateur $a\left(t, D_{t}\right)$ son polynôme caractéristique d'indéterminée $\lambda=\left(\lambda_{1}, \ldots, \lambda_{q}\right)$, défini par $\mathcal{P}(\lambda)=\sum_{\gamma \leq m} a_{\gamma} \prod_{i=1}^{q} \prod_{j=0}^{\gamma_{i}-1}\left(\lambda_{i}-j\right)$, où on convient que $\prod_{\phi}=1$. On obtient la relation

$$
a\left(t, D_{t}\right)=\mathcal{P}\left(t D_{t}\right)
$$

Nous ferons l'hypothèse suivante:

$$
\left\{\begin{array}{c}
\text { Il existe une constante } c_{0}>0 \text { telle que: } \\
|\mathcal{P}(\lambda)| \geq c_{0}|\lambda|^{|m|} \text { pour tout } \lambda \in \mathbb{N}^{q} \text { de longueur }|\lambda| \geq 1
\end{array}\right.
$$

On considère l'équation de Fuchs non linéaire suivante:

$$
\left\{\begin{array}{c}
a\left(t, D_{t}\right) u(t, x)=f(t, x, \mathcal{D} u(t, x)), \quad(t, x) \in \mathbb{C}^{q} \times \Omega, \\
u(0, x)=0, \quad x \in \Omega,
\end{array}\right.
$$

où $\mathcal{D} u=\left(D^{\beta} u\right)_{\beta \in B} ; D^{\beta} u=t^{\gamma} D_{t}^{\delta} D_{x}^{\alpha} u ; \beta=(\gamma, \delta, \alpha) \in B$ où $B$ est une partie finie de l'ensemble

$$
\mathcal{E}=\left\{(\gamma, \delta, \alpha) \in \mathbb{N}^{q} \times \mathbb{N}^{q} \times \mathbb{N}^{n} ; \quad|\gamma| \geq|\delta|, \quad|\delta|+d|\alpha| \leq|m| \quad \text { et } \quad|\alpha| \geq 1\right\}
$$

et $\Omega$ est un ouvert de $\mathbb{R}^{n}$ donné. Soit $\tau=\operatorname{card} B$; on pose $y=\left(y_{\beta}\right)_{\beta \in B} \in \mathbb{C}^{\tau}$ avec $y_{\beta}=D^{\beta} u$. Soit $\bar{b}_{0}=\min \{|\alpha| ; \quad(\gamma, \delta, \alpha) \in B\}$. On note $V$ un voisinage ouvert convexe de l'origine de $\mathbb{C}^{\tau}$. Nous supposerons que la nonlinéarité $f$ satisfait aux conditions suivantes:

(4) $\left\{\begin{array}{c}f(t, x, y) \text { est une fonction polynômiale par rapport à } x \text { dans } \Omega \text {, de degré } \\ \bar{b}<\bar{b}_{0} \text {, à coefficients holomorphes dans } \mathbb{C}^{q} \times V \text { vérifiant: }\end{array}\right.$

$$
\left\{\begin{array}{c}
f(0, x, 0)=\frac{\partial f}{\partial y_{\beta}}(0, x, 0)=0, \text { pour tout } x \in \Omega \text { et pour tout } \beta=(\gamma, \delta, \alpha) \\
\text { tel que }|\gamma|=|\delta| .
\end{array}\right.
$$

On a alors le théorème suivant.

Théorème 2.2. Sous les hypothèses (21), (4) et (5); l'équation (3) admet une unique solution $u \in G^{\omega,(d)}\left(\mathbb{C}^{q} \times \Omega\right)$.

\section{RÉDUCTION DU PROBLÈME}

Pour $\beta=(\gamma, \delta, \alpha) \in \mathbb{N}^{q} \times \mathbb{N}^{q} \times \mathbb{N}^{n}$ tel que $|\gamma|>|\delta|$, on écrit $D^{\beta} u=t^{\gamma-\gamma^{\prime}} D^{\beta^{\prime}} u$ où $D^{\beta^{\prime}} u=t^{\gamma^{\prime}} D_{t}^{\delta} D_{x}^{\alpha} u$, avec $\gamma^{\prime} \in \mathbb{N}^{q}$ est choisi tel que: $\gamma-\gamma^{\prime}>0$ et $\left|\gamma^{\prime}\right|=|\delta|$. 
Alors, en changeant de notation, on obtient $f(t, x, \mathcal{D} u)=f\left(t, x,\left(D^{\beta} u\right)_{\beta \in B}\right)$ où $\beta=(\gamma, \delta, \alpha) \in B$ avec $B$ une partie de l'ensemble:

$$
\mathcal{E}=\left\{(\gamma, \delta, \alpha) \in \mathbb{N}^{q} \times \mathbb{N}^{q} \times \mathbb{N}^{n} ; \quad|\gamma|=|\delta| \leq|m|-d|\alpha| \quad \text { et } \quad|\alpha| \geq 1\right\} .
$$

En utilisant la relation (11), le problème (3) s'écrit sous la forme

$$
\left\{\begin{array}{c}
\mathcal{P}\left(t D_{t}\right) u(t, x)=f(t, x, \mathcal{D} u(t, x)), \quad(t, x) \in \mathbb{C}^{q} \times \Omega, \\
u(0, x)=0, \quad x \in \Omega .
\end{array}\right.
$$

L'hypothèse (5) que vérifiait $f$ devient:

$$
f(0, x, 0)=\frac{\partial f}{\partial y_{\beta}}(0, x, 0)=0, \text { pour tout } x \in \Omega \text { et pour tout } \beta \in B .
$$

Pour tout $R>0$ et tout ouvert $\Omega$ de $\mathbb{R}^{n}$, on pose $D_{R}=\left\{t \in \mathbb{C}_{t}^{q} ; \max _{1 \leq i \leq q}\left|t_{i}\right|<R\right\}$ et $\Omega_{R}=D_{R} \times \Omega$. Le lemme 2.1 de [2] peut être formulé comme suit.

Lemme 3.3 (4]). Soient $R>0$ et $\Omega$ un ouvert de $\mathbb{R}^{n}$; alors l'opérateur $\mathcal{P}=$ $\mathcal{P}\left(t D_{t}\right)$ induit une bijection linéaire de l'espace des fonctions de $G^{\omega,(d)}\left(\Omega_{R}\right)$ qui s'annulent avec $t$ sur lui même et son inverse est défini par: $\left(\mathcal{P}^{-1} u\right)(t, x)=$ $\sum_{|k| \geq 1} \frac{t^{k}}{k !} \frac{D_{t}^{k} u(0, x)}{\mathcal{P}(k)}$. De plus, pour tout $(\delta, \alpha) \in \mathbb{N}^{q} \times \mathbb{N}^{n} ;$

$$
D_{t}^{\delta} D_{x}^{\alpha}\left(\mathcal{P}^{-1} u\right)(t, x)=\sum_{|k| \geq 1, k \geq \delta} \frac{t^{k-\delta}}{(k-\delta) !} \frac{D_{t}^{k} D_{x}^{\alpha} u(0, x)}{\mathcal{P}(k)} .
$$

Toutes ces séries convergent normalement sur tout compact de $\Omega_{R}$.

En remplaçant $u$ par $\mathcal{P}^{-1}(u)$ dans l'équation (7); alors pour tout $R>0$ et tout ouvert $\Omega^{\prime} \subset \Omega$, le problème (3) est dans $\Omega_{R}^{\prime}$ équivalent à l'équation réduite:

$$
\left\{\begin{array}{c}
u=f(t, x, \mathcal{H} u) \\
u=0, \quad \text { pour } t=0,
\end{array}\right.
$$

où $\mathcal{H} u=\left(\mathcal{H}_{\beta} u\right)_{\beta \in B} ; \mathcal{H}_{\beta} u=D^{\beta} \mathcal{P}^{-1}(u)$.

En tenant compte que $B \subset \mathcal{E}$ où $\mathcal{E}$ est défini par (6), alors le théorème 2.2 se déduira de la proposition suivante.

Proposition 3.4. Sous les hypothèses (2), (4) et (8); pour tout $R>0$, l'équation (9) admet une unique solution $u \in G^{\omega,(d)}\left(\Omega_{R}\right)$.

Le théorème 2.2 s'en déduit de la façon suivante: ayant assuré, par la proposition 3.4. l'existence et l'unicité de la solution de l'équation (9) dans $G^{\omega,(d)}\left(\Omega_{R}\right)$ pour tout $R>0$ alors, vu que $\mathbb{C}^{q}=\bigcup_{R>0} D_{R}$, on déduit d'aprés la définition 2.1 que l'équation (9) admet une unique solution $u$ dans $G^{\omega,(d)}\left(\mathbb{C}^{q} \times \Omega\right)$.

\section{Preuve de la proposition 3.4}

La résolution de l'équation (9) repose sur l'application du théorème du point fixe dans une algèbre de Banach associée à une fonction majorante. 
4.1. Cadre fonctionnel. On s'inspire du formalisme des fonctions majorantes développé dans [13. On utilise des séries formelles à deux indéterminées, notées $\sigma$ et $\xi$ où $\sigma=t_{1}+\ldots+t_{q}$ et $\xi=x_{1}+\ldots+x_{n}$. Soit $\phi(\sigma, \xi)=\sum_{j=0}^{+\infty} \frac{\xi^{j}}{j !} \phi_{j}(\sigma)$, on suppose que les séries entières $\phi_{j}$ sont toutes convergentes dans un polydisque $D_{R}$ où $R>0$. Étant donné un ouvert $\Omega$ de $\mathbb{R}^{n}$ et une fonction $u \in \mathcal{C}^{\omega, \infty}\left(\Omega_{R}\right)$, étant donné que $\frac{\xi^{j}}{j !}=\sum_{\alpha \in \mathbb{N}^{n},|\alpha|=j} \frac{x^{\alpha}}{\alpha !}$, on rappelle 13] que la relation $u \ll \phi$ signifie que

$$
\forall \alpha \in \mathbb{N}^{n}, \forall x \in \Omega, \quad D_{x}^{\alpha} u(t, x) \ll \phi_{|\alpha|}(\sigma),
$$

où cette dernière relation $\ll$ doit être comprise au sens des fonctions majorantes en $t$, ce qui signifie

$$
\forall \alpha \in \mathbb{N}^{n}, \forall x \in \Omega, \forall k \in \mathbb{N}^{q}, \quad\left|D_{t}^{k} D_{x}^{\alpha} u(0, x)\right| \leq D_{t}^{k} \phi_{|\alpha|}(0) .
$$

À la série formelle $\phi$, on associe la série formelle Gevrey

$$
\phi^{d}(\sigma, \xi)=\sum_{j=0}^{+\infty} \frac{\xi^{j}}{j !}(j !)^{d-1} \phi_{j}(\sigma) .
$$

Étant donné $R>0$ et un paramètre $\rho>0$, on considère la série formelle $\Phi_{\rho, R}^{d}(\sigma, \xi)=\sigma \phi_{\rho, R}^{d}(\sigma, \xi)$, où $\phi_{\rho, R}(\sigma, \xi) \in \mathbb{R}_{+}\{\sigma, \xi\}$ et est définie par

$$
\phi_{\rho, R}^{d}(\sigma, \xi)=\sum_{p=0}^{+\infty}(\rho \sigma)^{p}(\rho R)^{s^{\prime} p} \frac{\left(D^{s p} \varphi_{\rho R}\right)^{d}(\xi)}{(s p) !},
$$

$s$ est un entier donné tel que $s \geq \max (1,2|m|)$ et $s^{\prime}=s-1 \geq 0 . \varphi_{R}$ est la fonction majorante introduite dans [13] définie par $\varphi_{R}(\xi)=\frac{1}{L} \Theta(\xi / R)$ pour $R>0$ et, $\Theta$ est la fonction majorante de Lax [8] définie par $\Theta(\xi)=\sum_{j=0}^{+\infty} \frac{\xi^{j}}{(j+1)^{2}}, L$ est la constante de Lax qui vérifie $\Theta^{2} \ll L \Theta$ (au sens des séries majorantes).

Par conséquent, la fonction majorante $\varphi_{R}$ vérifie $\varphi_{R}^{2} \ll \varphi_{R}$ et on a $\varphi_{R}(0)=1 / L$. On écrit la série formelle (11) sous la forme

$$
\phi_{\rho, R}^{d}(\sigma, \xi)=\sum_{j=0}^{+\infty} \frac{\xi^{j}}{j !}(j !)^{d-1} \phi_{j, \rho, R}(\sigma),
$$

les séries entières $\phi_{j, \rho, R}$ ne dépendent que des paramètres $\rho$ et $R$ et on montre la

Proposition 4.5 (4]). Toutes les séries entières $\phi_{j, \rho, R}$ admettent $R$ pour rayon de convergence et, pour tout $0<r<R$, il existe des constantes $c_{1}=c_{1}(r, R) \geq 0$ et $c_{2}=2 / \rho R\left(1-(r / R)^{1 / s}\right)$ telles que:

$$
\max _{|\sigma| \leq r}\left|\phi_{j, \rho, R}(\sigma)\right| \leq c_{1} c_{2}^{j} j ! \text {, pour tout } j \in \mathbb{N} .
$$

La série (11) est donc bien de la forme voulue et cette série entière est convergente. Donc, $\phi_{\rho, R}^{d}$ est bien une fonction majorante, de plus elle vérifie

$$
\left(\phi_{\rho, R}^{d}\right)^{k} \ll \phi_{\rho, R}^{d}, \quad \text { pour tout entier } k \geq 1 \text { et pour tout } \rho, R>0 .
$$

On en déduit que l'espace de Banach (voir [13]), associé à $\phi_{\rho, R}^{d}$ défini par

$$
\mathcal{C}_{\phi_{\rho, R}}^{\omega, d}\left(\Omega_{R}\right)=\left\{u \in \mathcal{C}^{\omega, \infty}\left(\Omega_{R}\right) ; \text { il existe } c \geq 0 \text { tel que } u \ll c \phi_{\rho, R}^{d}\right\}
$$

muni de la norme $\min \left\{c \geq 0 ; u \ll c \phi_{\rho, R}^{d}\right\}$ qu'on note $\|\cdot\|_{\phi^{d}}$, est une algèbre de Banach. De même, on note $\mathcal{C}_{\Phi_{\rho, R}}^{\omega, d}\left(\Omega_{R}\right)$ et $\|\cdot\|_{\Phi^{d}}$ l'espace de Banach et la norme 
associés à la série formelle Gevrey $\Phi_{\rho, R}^{d}$. Dire que $u \in \mathcal{C}_{\Phi_{\rho, R}}^{\omega, d}\left(\Omega_{R}\right)$ signifie d'aprés (10) que $u \in \mathcal{C}^{\omega, \infty}\left(\Omega_{R}\right)$ et que:

$$
\forall \alpha \in \mathbb{N}^{n}, \forall x \in \Omega ; \quad D_{x}^{\alpha} u(t, x) \ll\|u\|_{\Phi^{d}}(|\alpha| !)^{d-1} \sigma \phi_{|\alpha|, \rho, R}(\sigma) .
$$

La propriété (12) nous permet d'établir la proposition 2.3 de [13] adaptée à la série formelle $\phi_{\rho, R}^{d}$.

Proposition $4.6\left([4)\right.$. Soient $u \in \mathcal{C}_{\phi_{\rho, R}}^{\omega, d}\left(\Omega_{R}\right)$ et $R_{1}>0$ tels que $\|u\|_{\phi^{d}}<R_{1}$, alors la fonction $\frac{R_{1}}{R_{1}-u}$ appartient à l'espace $\mathcal{C}_{\phi_{\rho, R}}^{\omega, d}\left(\Omega_{R}\right)$ et on a:

$$
\frac{R_{1}}{R_{1}-u} \ll\left(L+\frac{\|u\|_{\phi^{d}}}{R_{1}-\|u\|_{\phi^{d}}}\right) \phi_{\rho, R}^{d}(\sigma, \xi) .
$$

Pour les propriétés de la fonction majorante $\varphi_{\rho R}$ qui nous permettent de contrôler les opérateurs de dérivation, on s'inspire des lemmes 1.4 et 2.6 de [11] et on montre les deux lemmes suivants.

Lemme 4.7 ([4]). Pour tout $R, \rho>0$ et tout $i, j \in \mathbb{N}$ on a:

$$
D^{i} \varphi_{\rho R} \ll \frac{i !}{(i+j) !}(4 \rho R)^{j} D^{i+j} \varphi_{\rho R} .
$$

Lemme 4.8 (4]). Soit $p \in \mathbb{N}$ et soit $\nu$ le plus petit entier $\geq d p$, il existe une constante $c(p)=\frac{(\nu+1)^{2}}{(p+1)^{2}}>0$ telle que pour tout $R, \rho>0 ; D^{p}\left(D^{j} \varphi_{\rho R}\right)^{d} \ll c(p)(\rho R)^{\nu-p}$ $\left(D^{j+\nu} \varphi_{\rho R}\right)^{d}$, pour tout $j \in \mathbb{N}$.

Lemme 4.9. Soit $\eta>1$, alors il existe [13] une constante $c(\eta)>0$ telle que pour tout $\rho, R>0$;

$$
\frac{\eta \rho R}{\eta \rho R-\left(\left(\rho / 4^{s^{\prime}}\right) \sigma+\xi\right)} \ll c(\eta) \phi_{\rho, R}(\sigma, \xi) .
$$

Preuve. Soit $\eta>1$, alors d'aprés le lemme 2.4 de $[13]$ on a $\eta \rho R /(\eta \rho R-().) \ll$ $c(\eta) \varphi_{\rho R}($.$) , où$

$$
c(\eta)=\sup _{j \in \mathbb{N}} L(j+1)^{2} / \eta^{j} .
$$

Il suffit donc de montrer que: $\varphi_{\rho R}\left(\left(\rho / 4^{s^{\prime}}\right) \sigma+\xi\right) \ll \phi_{\rho, R}(\sigma, \xi)$.

D'aprés le lemme 4.7 et sachant que $s^{\prime}=s-1$ on obtient:

$$
\frac{D^{p} \varphi_{\rho R}(\xi)}{p !} \ll(4 \rho R)^{s^{\prime} p} \frac{D^{s p} \varphi_{\rho R}(\xi)}{(s p) !},
$$

d'où,

$$
\begin{gathered}
\varphi_{\rho R}\left(\left(\rho / 4^{s^{\prime}}\right) \sigma+\xi\right)=\sum_{p=0}^{+\infty}\left(\left(\rho / 4^{s^{\prime}}\right) \sigma\right)^{p} \frac{D^{p} \varphi_{\rho R}(\xi)}{p !} \\
\ll \sum_{p=0}^{+\infty}\left(\left(\rho / 4^{s^{\prime}}\right) \sigma\right)^{p}(4 \rho R)^{s^{\prime} p} \frac{D^{s p} \varphi_{\rho R}(\xi)}{(s p) !}=\phi_{\rho, R}(\sigma, \xi) .
\end{gathered}
$$

On rappelle que si $\beta=(\gamma, \delta, \alpha) \in B \subset \mathcal{E}$ alors $|\gamma|=|\delta| \leq|m|-d|\alpha|,|\alpha| \geq 1$ et $\mathcal{H}_{\beta} u=t^{\gamma} D_{t}^{\delta} D_{x}^{\alpha} \mathcal{P}^{-1}(u)$. On obtient le 
Lemme 4.10. Il existe une constante $c_{3}=c_{3}(|m|, s)>0$ telle que pour tout $R, \rho>0$, pour tout $a, a^{\prime}>0$ et $u, u^{\prime}$ vérifiant $u \ll a \Phi_{\rho, R}^{d}$ et $u^{\prime} \ll a^{\prime} \Phi_{\rho, R}^{d}$ on a: pour tout $\beta=(\gamma, \delta, \alpha) \in B, \beta^{\prime}=\left(\gamma^{\prime}, \delta^{\prime}, \alpha^{\prime}\right) \in B$

$$
\begin{gathered}
\mathcal{H}_{\beta} u \ll c_{3} \rho^{-|\alpha|} R^{1-|\alpha|} a \phi_{\rho, R}^{d}(\sigma, \xi), \\
\mathcal{H}_{\beta} u \mathcal{H}_{\beta^{\prime}} u^{\prime} \ll c_{3} \rho^{-|\alpha|-\left|\alpha^{\prime}\right|} R^{\left(1-|\alpha|-\left|\alpha^{\prime}\right|\right)} \text { a } a^{\prime} \Phi_{\rho, R}^{d}(t, x) .
\end{gathered}
$$

Preuve. Soient $R, \rho>0$ fixés et $\beta=(\gamma, \delta, \alpha) \in B, \beta^{\prime}=\left(\gamma^{\prime}, \delta^{\prime}, \alpha^{\prime}\right) \in B$.

D'aprés le lemme 3.3

$$
\mathcal{H}_{\beta} u(t, x)=\sum_{|k| \geq 1, k \geq \delta} \frac{t^{k-\delta+\gamma}}{(k-\delta) !} \frac{D_{x}^{\alpha} D_{t}^{k} u(0, x)}{\mathcal{P}(k)} .
$$

Soit $a>0$ et supposons que $u \ll a \Phi_{\rho, R}^{d}$. Alors, $D_{x}^{\alpha} D_{t}^{k} u(0, x) \ll a D_{x}^{\alpha} D_{t}^{k} \Phi_{\rho, R}^{d}(0, x)$. En utilisant (11) on obtient:

$$
D_{x}^{\alpha} D_{t}^{k} \Phi_{\rho, R}^{d}(0, x)=\rho^{|k|-1}|k| !(\rho R)^{s^{\prime}(|k|-1)} \frac{D_{x}^{\alpha}\left(D^{s(|k|-1)} \varphi_{\rho R}\right)^{d}(\xi)}{[s(|k|-1)] !} .
$$

Comme pour $(\gamma, \delta, \alpha) \in B$ on a $|m|-|\delta| \geq d|\alpha|$, alors d'aprés le lemme 4.8 il existe $c=c(|\alpha|)>0$ tel que

$$
\begin{aligned}
& D_{x}^{\alpha} D_{t}^{k} u(0, x) \\
& \ll c a(\rho R)^{(|m|-|\delta|-|\alpha|)}\left(\rho^{|k|-1}|k| !(\rho R)^{s^{\prime}(|k|-1)} \frac{D^{s(|k|-1)+|m|-|\delta|} \varphi_{\rho R}(\xi)}{[s(|k|-1)] !}\right)^{d} .
\end{aligned}
$$

Notons:

$\mathcal{F}_{\beta}(t, \xi)=\sum_{|k| \geq 1, k \geq \delta} \rho^{|k|-1}|k| ! \frac{t^{k-\delta+\gamma}}{(k-\delta) !} \frac{1}{|\mathcal{P}(k)|}(\rho R)^{s^{\prime}(|k|-1)} \frac{D^{s(|k|-1)+|m|-|\delta|} \varphi_{\rho R}(\xi)}{[s(|k|-1)] !}$,

alors

$$
\mathcal{H}_{\beta} u(t, x) \ll c a(\rho R)^{(|m|-|\delta|-|\alpha|)}\left(\mathcal{F}_{\beta}\right)^{d}(t, \xi) .
$$

En appliquant le lemme 4.7 on obtient

$$
\begin{aligned}
& \mathcal{F}_{\beta}(t, \xi) \ll \sum_{|k| \geq 1, k \geq \delta} \quad\left[\rho^{|k|-1}|k| ! \frac{t^{k-\delta+\gamma}}{(k-\delta+\gamma) !} \frac{(k-\delta+\gamma) !}{(k-\delta) !} \frac{1}{|\mathcal{P}(k)|}(\rho R)^{s^{\prime}(|k|-1)}\right. \\
& \left.\frac{(s(|k|-1)+|m|-|\delta|) !}{[s(|k|-1)] !}(4 \rho R)^{|\delta|} \frac{D^{s(|k|-1)+|m|} \varphi_{\rho R}(\xi)}{[s(|k|-1)+|m|] !}\right] .
\end{aligned}
$$

En utilisant l'inégalité $\frac{k !}{k^{\prime} !} \leq \frac{|k| !}{\left|k^{\prime}\right| !}$ pour tout $k, k^{\prime} \in \mathbb{N}^{q}$ tels que $k \geq k^{\prime}$ et, vu l'hypothèse (2) on obtient l'existence d'une constante $c=c(|m|, s)>0$ telle que pour tout $|k| \geq 1$,

$\frac{(k-\delta+\gamma) !}{(k-\delta) !} \frac{1}{|\mathcal{P}(k)|} \frac{(s(|k|-1)+|m|-|\delta|) !}{[s(|k|-1)] !} \leq \frac{1}{c_{0}}\left(\frac{s(|k|-1)+|m|}{|k|}\right)^{|m|-|\delta|} \leq \frac{c}{c_{0}}$.

Sachant que $|\gamma|=|\delta|$ et en utilisant la relation

$$
\sum_{k \in \mathbb{N}^{q},|k|=p}\left(\rho^{|k|} t^{k}\right) / k !=(\rho \sigma)^{p} / p !
$$

on obtient

$$
\mathcal{F}_{\beta}(t, \xi) \ll \frac{c}{c_{0}} \rho^{-1}(4 \rho R)^{|\delta|} \sum_{p=0}^{+\infty}(\rho \sigma)^{p+1}(\rho R)^{s^{\prime} p} \frac{D^{s p+|m|} \varphi_{\rho R}(\xi)}{(s p+|m|) !} .
$$


Comme $s \geq|m|$, alors en appliquant le lemme 4.7 et en tenant compte que $s-s^{\prime}=1$, on obtient

$$
\begin{aligned}
\mathcal{F}_{\beta}(t, \xi) & \ll \frac{c}{c_{0}} 4^{s+|\delta|-|m|} \rho^{|\delta|-|m|} R^{1+|\delta|-|m|} \sum_{p=0}^{+\infty}(\rho \sigma)^{p+1}(\rho R)^{s^{\prime}(p+1)} \frac{D^{s(p+1)} \varphi_{\rho R}(\xi)}{(s(p+1)) !} \\
& \ll \frac{c}{c_{0}} 4^{s} \rho^{|\delta|-|m|} R^{1+|\delta|-|m|} \phi_{\rho, R}(\sigma, \xi) .
\end{aligned}
$$

En utilisant le lemme 2.3-1 de [1] et en reportant le résultat dans (14), on déduit qu'il existe $c_{3}=c_{3}(|m|, s)>0$ tel que $\mathcal{H}_{\beta} u(t, x) \ll c_{3} \rho^{-|\alpha|} R^{(1-|\alpha|)} a \phi_{\rho, R}^{d}(\sigma, \xi)$.

D'autre part, pour $u, u^{\prime}$ vérifiant $u \ll a \Phi_{\rho, R}^{d}$ et $u^{\prime} \ll a^{\prime} \Phi_{\rho, R}^{d}$, en appliquant le lemme 2.3-2 de [11, on écrit d'aprés (14),

$$
\begin{aligned}
& \mathcal{H}_{\beta} u(t, x) \mathcal{H}_{\beta^{\prime}} u^{\prime}(t, x) \\
& \quad \ll a a^{\prime} c(|\alpha|) c\left(\left|\alpha^{\prime}\right|\right)(\rho R)^{2|m|-\left(|\delta|+\left|\delta^{\prime}\right|\right)-\left(|\alpha|+\left|\alpha^{\prime}\right|\right)}\left(\mathcal{F}_{\beta} \mathcal{F}_{\beta^{\prime}}\right)^{d}(t, \xi),
\end{aligned}
$$

En notant $b_{j}=D^{j} \varphi_{\rho R} / j$ ! alors vu (15), il existe $c^{\prime}=c^{\prime}(|m|, s)>0$ tel que

$$
\left(\mathcal{F}_{\beta} \mathcal{F}_{\beta^{\prime}}\right)(t, \xi) \ll c^{\prime} /\left(c_{0}\right)^{2}(4 \rho R)^{|\delta|+\left|\delta^{\prime}\right|} \sigma^{2} \sum_{p=0}^{+\infty}(\rho \sigma)^{p}(\rho R)^{s^{\prime} p}\left(\sum_{j=0}^{p} b_{s j+|m|} b_{s(p-j)+|m|}\right) .
$$

Comme $s \geq 2|m|$ alors en appliquant le lemme 4.7 et en dérivant l'inégalité $\varphi_{\rho R}^{2} \ll$ $\varphi_{\rho R}$ à l'ordre $s(p+1)$ on montre que $\sum_{j=0}^{p} b_{s j+|m|} b_{s(p-j)+|m|} \ll(4 \rho R)^{s-2|m|} b_{s(p+1)}$. Ainsi, sachant que $s-s^{\prime}=1$, on obtient:

$$
\begin{aligned}
\left(\mathcal{F}_{\beta} \mathcal{F}_{\beta^{\prime}}\right)(t, \xi) & \ll c^{\prime} /\left(c_{0}\right)^{2} 4^{s}(4 \rho R)^{|\delta|+\left|\delta^{\prime}\right|-2|m|} R \sigma \sum_{p=0}^{+\infty}(\rho \sigma)^{p+1}(\rho R)^{s^{\prime}(p+1)} b_{s(p+1)} \\
& \ll \frac{c^{\prime}}{\left(c_{0}\right)^{2}} 4^{s}(\rho R)^{|\delta|+\left|\delta^{\prime}\right|-2|m|} R \Phi_{\rho, R}(t, x) .
\end{aligned}
$$

En appliquant le lemme 2.3-1 de [11] et en reportant le résultat dans (16) on déduit le résultat voulu.

Si $V_{1}$ et $V_{2}$ sont deux voisinages de l'origine de $\mathbb{C}^{\tau}$, par extension on note $\mathcal{C}^{\omega, \infty}\left(U \times \Omega \times V_{1} \times V_{2}\right)$ l'algèbre des fonctions $F(t, x, y, z)$ holomorphes en $(t, y, z) \in$ $U \times V_{1} \times V_{2}$ et indéfiniment différentiables en $x \in \Omega$.

Lemme 4.11. Étant donné $R>0, \eta>1$, considérons

$$
F(t, x, y, z) \in \mathcal{C}^{\omega, \infty}\left(\Omega_{4^{s^{\prime} \eta R}} \times \triangle_{R_{1}} \times \triangle_{R_{2}}\right),
$$

où pour $R_{1}, R_{2}>0$ on note $\triangle_{R_{1}}=\left\{y \in \mathbb{C}^{\tau} ; \max _{1 \leq k \leq \tau}\left|y_{k}\right|<R_{1}\right\}$ et $\triangle_{R_{2}}=\{z \in$ $\left.\mathbb{C}^{\tau} ; \max _{1 \leq k \leq \tau}\left|z_{k}\right|<R_{2}\right\}$. S'il existe un entier $\bar{q} \in \mathbb{N}$ et une constante $M \geq 0$ tels que, pour tout $(t, x, y, z) \in \Omega_{4^{s^{\prime} \eta R}} \times \triangle_{R_{1}} \times \triangle_{R_{2}}$;

$$
\left|D_{x}^{\alpha} F(t, x, y, z)\right| \leq\left\{\begin{array}{ccc}
M & \text { si } & |\alpha| \leq \bar{q}, \\
0 & \text { si } & |\alpha|>\bar{q},
\end{array}\right.
$$

alors il existe une constante $c(\eta)>0$ telle que pour tout $\rho>0$ vérifiant $\rho R>1$ on a, pour tout $(t, x, y, z) \in \Omega_{4^{\prime} \eta R} \times \triangle_{R_{1}} \times \triangle_{R_{2}}$,

$$
F(t, x, y, z) \ll c(\eta) M(\eta \rho R)^{\bar{b}} \phi_{\rho, R}^{d}(\sigma, \xi) \prod_{k=1}^{\tau} \frac{R_{1}}{R_{1}-y_{k}} \prod_{k=1}^{\tau} \frac{R_{2}}{R_{2}-z_{k}} .
$$


Proof. Soient $R>0, \eta>1$ fixés. Soit $\rho>0$ tel que $\rho R>1$, alors $1 \leq$ $(\eta \rho R)^{\bar{q}}\left(\frac{1}{\eta \rho R}\right)^{|\alpha|}(|\alpha| !)^{d}$ si $|\alpha| \leq \bar{q}$. Ainsi, d'aprés les inégalités de Cauchy, pour tout $(t, x, y, z) \in \Omega_{4^{s^{\prime} \eta R}} \times \triangle_{R_{1}} \times \triangle_{R_{2}}$ on a:

$$
\begin{aligned}
& D_{x}^{\alpha} F(t, x, y, z) \\
& \qquad \begin{cases}M(\eta \rho R)^{\bar{q}}\left(\frac{1}{\eta \rho R}\right)^{|\alpha|}(|\alpha| !)^{d} \frac{4^{s^{\prime}} \eta R}{4^{\prime} \eta R-\sigma} \prod_{k=1}^{\tau} \frac{R_{1}}{R_{1}-y_{k}} \frac{R_{2}}{R_{2}-z_{k}} & \text { si }|\alpha| \leq \bar{q}, \\
0 & \text { si }|\alpha|>\bar{q},\end{cases}
\end{aligned}
$$

ce qui signifie exactement au sens des séries majorantes (définition (10)), que

$F(t, x, y, z)$

$$
\begin{aligned}
& \ll M(\eta \rho R)^{\bar{q}} \sum_{j=0}^{\bar{q}} \frac{(\xi / \eta \rho R)^{j}}{j !}(j !)^{d} \frac{4^{s^{\prime}} \eta R}{4^{s^{\prime}} \eta R-\sigma} \prod_{k=1}^{\tau} \frac{R_{1}}{R_{1}-y_{k}} \prod_{k=1}^{\tau} \frac{R_{2}}{R_{2}-z_{k}} \\
& \ll M(\eta \rho R)^{\bar{q}} \sum_{j=0}^{+\infty}(\xi / \eta \rho R)^{j}(j !)^{d-1} \frac{\eta \rho R}{\eta \rho R-\left(\rho / 4^{s^{\prime}}\right) \sigma} \prod_{k=1}^{\tau} \frac{R_{1}}{R_{1}-y_{k}} \prod_{k=1}^{\tau} \frac{R_{2}}{R_{2}-z_{k}} \\
& =M(\eta \rho R)^{\bar{q}}\left(\sum_{j=0}^{+\infty}(\xi / \eta \rho R)^{j} \frac{\eta \rho R}{\eta \rho R-\left(\rho / 4^{s^{\prime}}\right) \sigma}\right) \prod_{k=1}^{\tau} \frac{R_{1}}{R_{1}-y_{k}} \prod_{k=1}^{\tau} \frac{R_{2}}{R_{2}-z_{k}} .
\end{aligned}
$$

Or,

$$
\begin{aligned}
\sum_{j=0}^{+\infty}(\xi / \eta \rho R)^{j} \frac{\eta \rho R}{\eta \rho R-\left(\rho / 4^{s^{\prime}}\right) \sigma} & =\frac{1}{1-(\xi / \eta \rho R)} \frac{\eta \rho R}{\eta \rho R-\left(\rho / 4^{s^{\prime}}\right) \sigma} \\
& \ll \frac{\eta \rho R}{\eta \rho R-\left(\left(\rho / 4^{s^{\prime}}\right) \sigma+\xi\right)} .
\end{aligned}
$$

On obtient le résultat voulu en utilisant le lemme 4.9 et le lemme 2.3-1 de [11].

Quant aux fonctions $f(t, x, 0)$ et $\frac{\partial f}{\partial y_{\beta}}(t, x, 0)$, on en déduit le corollaire suivant.

Corollaire 4.12. Étant donné $R>0, \eta>1$ et $\Omega^{\prime}$ un ouvert relativement compact de $\Omega$; il existe une constante $M_{f}=M_{f}\left(\bar{b}, \Omega^{\prime}, \eta, R, f\right) \geq 0$ telle que pour tout $\rho>0$ vérifiant $\rho R>1$ on a: pour tout $(t, x) \in D_{4^{s^{\prime}} \eta R} \times \Omega^{\prime} ; \quad f(t, x, 0), \frac{\partial f}{\partial y_{\beta}}(t, x, 0) \ll$ $M_{f} \rho^{\bar{b}} \Phi_{\rho, R}^{d}(t, x)$.

4.2. Existence de la solution de l'équation (9) dans $G^{\omega,(d)}\left(\Omega_{R}\right)$. Étant donné que $f(t, x, y)$ vérifie (8), on écrit:

$$
f(t, x, y)=f(t, x, 0)+\sum_{\beta \in B} \frac{\partial f}{\partial y_{\beta}}(t, x, 0) y_{\beta}+g(t, x, y), \quad \text { où } y=\left(y_{\beta}\right)_{\beta \in B}
$$

et $g$ est une fonction polynômiale par rapport à $x$ dans $\Omega$, de degré inférieur ou égal à $\bar{b}$, à coefficients holomorphes dans $\mathbb{C}^{q} \times V$ et vérifie $g(t, x, 0)=\frac{\partial g}{\partial y_{\beta}}(t, x, 0) \equiv 0$. On peut alors écrire:

$$
\begin{gathered}
g(t, x, y)-g\left(t, x, y^{\prime}\right) \\
=\sum_{\beta, \beta^{\prime} \in B}\left[F_{\beta, \beta^{\prime}}\left(t, x, y, y^{\prime}\right) y_{\beta}\left(y_{\beta^{\prime}}-y_{\beta^{\prime}}^{\prime}\right)+K_{\beta, \beta^{\prime}}\left(t, x, y, y^{\prime}\right) y_{\beta^{\prime}}^{\prime}\left(y_{\beta}-y_{\beta}^{\prime}\right)\right],
\end{gathered}
$$


où $F_{\beta, \beta^{\prime}}$ et $K_{\beta, \beta^{\prime}}$ sont des fonctions polynômiales par rapport à $x$ dans $\Omega$, de degré inférieur ou égal à $\bar{b}$, à coefficients holomorphes dans $\mathbb{C}^{q} \times V \times V$. On considère l'application:

$$
\begin{gathered}
\mathcal{F}: u \longmapsto f(t, x, 0)+\sum_{\beta \in B} \frac{\partial f}{\partial y_{\beta}}(t, x, 0) \mathcal{H}_{\beta} u+g(t, x, \mathcal{H} u), \\
\text { où on pose } y=\left(y_{\beta}\right)_{\beta \in B}=\left(\mathcal{H}_{\beta} u\right)_{\beta \in B}=\mathcal{H} u .
\end{gathered}
$$

Soient $R>0$ fixé et $\rho>0$ qui vérifie $\rho R>1$. Étant donné $\eta>1$ et $\Omega^{\prime}$ un ouvert relativement compact dans $\Omega$, on a d'aprés le corollaire 4.12 $\mathcal{F}(0)=$ $f(t, x, 0) \in \mathcal{C}_{\Phi_{\rho, R}}^{\omega, d}\left(\Omega_{R}^{\prime}\right)$ et $\|\mathcal{F}(0)\|_{\Phi^{d}}=\|f(t, x, 0)\|_{\Phi^{d}} \leq M_{f} \rho^{\bar{b}}$, où $M_{f}$ est une constante indépendante de $\rho$. Posons

$$
a_{\rho}=2 M_{f} \rho^{\bar{b}} .
$$

On démontre la proposition de contraction suivante.

Proposition 4.13. Pour tout $R>0$ et tout ouvert $\Omega^{\prime}$ relativement compact dans $\Omega$, il existe $\rho_{0}>0$ tel que pour tout $\rho \geq \rho_{0}$; l'application $\mathcal{F}$ est une contraction stricte de la boule fermée $B\left(0, a_{\rho}\right)$ de l'espace de Banach $\mathcal{C}_{\Phi_{\rho, R}}^{\omega, d}\left(\Omega_{R}^{\prime}\right) ; B\left(0, a_{\rho}\right)=$ $\left\{u \in \mathcal{C}_{\Phi_{\rho, R}}^{\omega, d}\left(\Omega_{R}^{\prime}\right) ; \quad\|u\|_{\Phi^{d}} \leq a_{\rho}\right\}$.

Preuve. Soient $R>0, \eta>1$ et $\Omega^{\prime}$ un ouvert relativement compact dans $\Omega$ fixés. Soit $\rho>0$ vérifiant $\rho R>1$. Considèrons $u, u^{\prime} \in B\left(0, a_{\rho}\right) \subset \mathcal{C}_{\Phi_{\rho, R}}^{\omega, d}\left(\Omega_{R}^{\prime}\right)$. Alors, $u \ll\|u\|_{\Phi^{d}} \Phi_{\rho, R}^{d} \ll a_{\rho} \Phi_{\rho, R}^{d}$ et $u^{\prime} \ll\left\|u^{\prime}\right\|_{\Phi^{d}} \Phi_{\rho, R}^{d} \ll a_{\rho} \Phi_{\rho, R}^{d}$.

Considèrons $\beta=(\gamma, \delta, \alpha) \in B, \beta^{\prime}=\left(\gamma^{\prime}, \delta^{\prime}, \alpha^{\prime}\right) \in B$ et posons:

$$
y=\left(y_{\beta}\right)_{\beta \in B}=\left(\mathcal{H}_{\beta} u\right)_{\beta \in B}=\mathcal{H} u, \quad y^{\prime}=\left(y_{\beta}^{\prime}\right)_{\beta \in B}=\left(\mathcal{H}_{\beta} u^{\prime}\right)_{\beta \in B}=\mathcal{H} u^{\prime} .
$$

On a

$$
\mathcal{F}(u)-\mathcal{F}\left(u^{\prime}\right)=\sum_{\beta \in B} \frac{\partial f}{\partial y_{\beta}}(t, x, 0)\left(y_{\beta}-y_{\beta}^{\prime}\right)+g(t, x, y)-g\left(t, x, y^{\prime}\right) .
$$

En utilisant le lemme 4.10 et le corollaire 4.12 et, vu que $\phi_{\rho, R}^{d}(\sigma, \xi) \Phi_{\rho, R}^{d}(\sigma, \xi) \ll$ $\Phi_{\rho, R}^{d}(\sigma, \xi)$, on obtient pour tout $(t, x) \in \Omega_{R}^{\prime} \subset \Omega_{4^{\prime} \eta R}^{\prime} ;$

$$
\sum_{\beta \in B} \frac{\partial f}{\partial y_{\beta}}(t, x, 0)\left(y_{\beta}-y_{\beta}^{\prime}\right) \ll \varepsilon_{1}\left(\frac{1}{\rho}\right)\left\|u-u^{\prime}\right\|_{\Phi^{d}} \Phi_{\rho, R}^{d}(\sigma, \xi)
$$

où $\varepsilon_{1}\left(\frac{1}{\rho}\right)=c_{3} M_{f}\left(\sum_{\beta \in B} R^{1-|\alpha|} \rho^{\bar{b}-|\alpha|}\right)$.

D'autre part, d'aprés le lemme 4.10 il existe $c_{3}=c_{3}(|m|, s)>0$ tel que

$$
y_{\beta}, y_{\beta}^{\prime} \ll c_{3} \rho^{-|\alpha|} R^{1-|\alpha|} a_{\rho} \phi_{\rho, R}^{d}(\sigma, \xi)=2 c_{3} M_{f} R^{1-|\alpha|} \rho^{\bar{b}-|\alpha|} \phi_{\rho, R}^{d}(\sigma, \xi) .
$$

Soit $R_{1}>0$ tel que

$$
\triangle_{R_{1}}=\left\{y \in \mathbb{C}^{\tau} ; \quad \max _{\beta \in B}\left|y_{\beta}\right|<R_{1}\right\} \subset V,
$$

comme pour tout $\beta=(\gamma, \delta, \alpha) \in B$ on a $\bar{b}<|\alpha|$, alors on choisit $\rho>0$ suffisamment grand (donc il existe $\rho_{0}>0$ tel que $\rho \geq \rho_{0}$ ) pour que $\rho R>1$ soit vérifié et tel que

$$
2 c_{3} M_{f} R^{1-|\alpha|} \rho^{\bar{b}-|\alpha|} \leq R_{1} / 2,
$$


où $R_{1}>0$ vérifie (19). Alors, pour $\rho$ suffisamment grand $\left(\rho \geq \rho_{0}\right)$ on obtient $\frac{\left\|y_{\beta}\right\|_{\phi^{d}}}{R_{1}-\left\|y_{\beta}\right\|_{\phi^{d}}}<1$ et $\frac{\left\|y_{\beta}^{\prime}\right\|_{\phi^{d}}}{R_{1}-\left\|y_{\beta}^{\prime}\right\|_{\phi^{d}}}<1$ et donc d'après la proposition 4.6 .

$$
\frac{R_{1}}{R_{1}-y_{\beta}}, \frac{R_{1}}{R_{1}-y_{\beta}^{\prime}} \ll(L+1) \phi_{\rho, R}^{d}(\sigma, \xi) .
$$

Lorsque $\beta, \beta^{\prime} \in B$, les fonctions $F_{\beta, \beta^{\prime}}$ et $K_{\beta, \beta^{\prime}}$ vérifient le lemme 4.11 avec $\bar{q}=\bar{b}$, $\Omega^{\prime}$ au lieu de $\Omega$ et

$$
M=\max _{\beta, \beta^{\prime} \in B} \sup \left\{\left|D_{x}^{\alpha} L_{\beta, \beta^{\prime}}\left(t, x, y, y^{\prime}\right)\right| ; \quad\left(t, x, y, y^{\prime}\right) \in \Omega_{4^{s^{\prime}} \eta R}^{\prime} \times\left(\triangle_{R_{1}}\right)^{2} \text { et }|\alpha| \leq \bar{b}\right\},
$$

où $L_{\beta, \beta^{\prime}}$ est l'une des fonctions $F_{\beta, \beta^{\prime}}$ ou $K_{\beta, \beta^{\prime}}, M=M\left(\bar{b}, \Omega^{\prime}, \eta, R, R_{1}, f\right) \geq 0$ est une constante indépendante de $\rho$.

Ainsi, pour tout $(t, x) \in \Omega_{R}^{\prime} \subset \Omega_{4^{s^{\prime}} \eta R}^{\prime}$, pour $\rho$ suffisamment grand $\left(\rho \geq \rho_{0}\right)$ et vu (12) on obtient:

$$
F_{\beta, \beta^{\prime}}\left(t, x, y, y^{\prime}\right), K_{\beta, \beta^{\prime}}\left(t, x, y, y^{\prime}\right) \ll c(\eta) M(\eta \rho R)^{\bar{b}}(L+1)^{2 \tau} \phi_{\rho, R}^{d}(\sigma, \xi) .
$$

D'aprés le lemme 4.10, il existe $c_{3}=c_{3}(|m|, s)>0$ tel que:

$y_{\beta}\left(y_{\beta^{\prime}}-y_{\beta^{\prime}}^{\prime}\right), \quad y_{\beta^{\prime}}^{\prime}\left(y_{\beta}-y_{\beta}^{\prime}\right) \ll c_{3} R^{1-|\alpha|-\left|\alpha^{\prime}\right|} \rho^{-|\alpha|-\left|\alpha^{\prime}\right|} a_{\rho}\left\|u-u^{\prime}\right\|_{\Phi^{d}} \Phi_{\rho, R}^{d}(\sigma, \xi)$, en combinant cette majoration avec (21) et en reportant le résultat dans (17), on obtient pour $\rho$ suffisamment grand $\left(\rho \geq \rho_{0}\right)$ :

$$
g(t, x, y)-g\left(t, x, y^{\prime}\right) \ll \varepsilon_{2}\left(\frac{1}{\rho}\right)\left\|u-u^{\prime}\right\|_{\Phi^{d}} \Phi_{\rho, R}^{d}(\sigma, \xi)
$$

où

$$
\varepsilon_{2}\left(\frac{1}{\rho}\right)=4 c_{3} M M_{f} \eta^{\bar{b}} c(\eta)(L+1)^{2 \tau}\left(\sum_{\beta, \beta^{\prime} \in B^{\prime}} R^{\bar{b}+1-|\alpha|-\left|\alpha^{\prime}\right|} \rho^{2 \bar{b}-|\alpha|-\left|\alpha^{\prime}\right|}\right) .
$$

De (18) et (22), on déduit que pour $\rho$ assez grand $\left(\rho \geq \rho_{0}\right)$;

$$
\mathcal{F}(u)-\mathcal{F}\left(u^{\prime}\right) \ll\left(\varepsilon_{1}\left(\frac{1}{\rho}\right)+\varepsilon_{2}\left(\frac{1}{\rho}\right)\right)\left\|u-u^{\prime}\right\|_{\Phi^{d}} \Phi_{\rho, R}^{d}(\sigma, \xi) .
$$

Comme $\bar{b}<|\alpha|$ et $\bar{b}<\left|\alpha^{\prime}\right|$, on choisit $\rho$ assez grand $\left(\rho \geq \rho_{0}\right)$ tel que les conditions: $\rho R>1$, (19) et (20) soient vérifiées et tel que: $\left(\varepsilon_{1}\left(\frac{1}{\rho}\right)+\varepsilon_{2}\left(\frac{1}{\rho}\right)\right) \leq \frac{1}{2}$. Ainsi, pour $\rho$ assez grand $\left(\rho \geq \rho_{0}\right)$, pour $u, u^{\prime} \in B\left(0, a_{\rho}\right) \subset \mathcal{C}_{\Phi_{\rho, R}}^{\omega, d}\left(\Omega_{R}^{\prime}\right)$ on a

$$
\mathcal{F}(u)-\mathcal{F}\left(u^{\prime}\right) \ll \frac{1}{2}\left\|u-u^{\prime}\right\|_{\Phi^{d}} \Phi_{\rho, R}^{d}(t, x) .
$$

D'autre part, $\mathcal{F}(u)=\mathcal{F}(u)-\mathcal{F}(0)+\mathcal{F}(0)$ et $\mathcal{F}(0)=f(t, x, 0) \in \mathcal{C}_{\Phi_{\rho, R}}^{\omega, d}\left(\Omega_{R}^{\prime}\right)$, donc $\mathcal{F}(u) \in \mathcal{C}_{\Phi \rho, R}^{\omega, d}\left(\Omega_{R}^{\prime}\right)$ et de plus,

$$
\|\mathcal{F}(u)\|_{\Phi^{d}} \leq \frac{1}{2}\|u\|_{\Phi^{d}}+\|\mathcal{F}(0)\|_{\Phi^{d}} \leq \frac{1}{2} a_{\rho}+\|\mathcal{F}(0)\|_{\Phi^{d}} \leq \frac{1}{2} a_{\rho}+\frac{1}{2} a_{\rho} .
$$

On déduit que $\mathcal{F}(u) \in B\left(0, a_{\rho}\right)$ et $\mathcal{F}$ est une contraction stricte de la boule $B\left(0, a_{\rho}\right)$ de $\mathcal{C}_{\Phi_{\rho, R}}^{\omega, d}\left(\Omega_{R}^{\prime}\right)$.

Grâce au théorème du point fixe de Banach on obtient la 
Proposition 4.14. Pour tout $R>0$ et tout ouvert $\Omega^{\prime}$ relativement compact dans $\Omega$ fixés, il existe $\rho_{0}>0$ tel que pour tout $\rho \geq \rho_{0}$; l'application $\mathcal{F}$ admet un point fixe unique dans $\mathcal{C}_{\Phi_{\rho, R}}^{\omega, d}\left(\Omega_{R}^{\prime}\right)$.

Lemme 4.15. Pour tout $R>0$, l'équation (9) admet au moins une solution $u \in G^{\omega,(d)}\left(\Omega_{R}\right)$.

Preuve. Fixons $R>0$. Soient $\Omega^{\prime \prime}$ un compact de $\Omega$ et $h>0$. $\Omega$ étant la réunion de tous les ouverts relativement compacts de $\Omega$, il existe $i_{0} \in \mathbb{N}$ tel que $\Omega^{\prime \prime} \subset \Omega_{i_{0}}^{\prime}$. D'aprés la proposition 4.14 il existe $\rho_{i_{0}}=\rho_{\Omega_{i_{0}}^{\prime}}>0$ tel que l'équation (9) admette une unique solution dans $\mathcal{C}_{\Phi_{\rho . R}}^{\omega, d}\left(D_{R} \times \Omega_{i_{0}}^{\prime}\right)$ pour tout $\rho \geq \rho_{i_{0}}$. En notant $\|u\|_{\Phi^{d}}^{(\rho)}$ sa norme on obtient d'aprés (13), pour tout $\rho \geq \rho_{i_{0}}$;

$$
\forall \alpha \in \mathbb{N}^{n}, \forall x \in \Omega_{i_{0}}^{\prime} ; \quad D_{x}^{\alpha} u(t, x) \ll\|u\|_{\Phi^{d}}^{(\rho)}(|\alpha| !)^{d-1} \sigma \phi_{|\alpha|, \rho, R}(\sigma) .
$$

D'aprés la proposition 4.5. pour tout $r \in] 0, R\left[\right.$, pour tout $t \in \bar{U}_{r}$ et tout $x \in \Omega^{\prime \prime} \subset$ $\Omega_{i_{0}}^{\prime}$ et, vu que $|\alpha| ! \leq 2^{|\alpha|} \alpha$ ! on obtient

$$
\forall \alpha \in \mathbb{N}^{n} ;\left|D_{x}^{\alpha} u(t, x)\right| \leq r c_{2}\|u\|_{\Phi^{d}}^{(\rho)}\left(2^{d+1} / \rho R\left(1-(r / R)^{1 / s}\right)\right)^{|\alpha|}(\alpha !)^{d} .
$$

On choisit $\rho$ suffisamment grand $\left(\rho \geq \rho_{i_{0}}\right)$ tel que: $2^{d+1} / \rho R\left(1-(r / R)^{1 / s}\right)<h$. Alors, pour tout $r>0$ tel que $\bar{D}_{r} \subset D_{R}$ et tout compact $\Omega^{\prime \prime}$ de $\Omega$; il existe $c=r c_{2}\|u\|_{\Phi^{d}}^{(\rho)}$ tel que

$$
\forall \alpha \in \mathbb{N}^{n}, \forall(t, x) \in \bar{D}_{r} \times \Omega^{\prime \prime} ; \quad\left|D_{x}^{\alpha} u(t, x)\right| \leq c h^{|\alpha|}(\alpha !)^{d} .
$$

4.3. Unicité de la solution de l'équation (9) dans $G^{\omega,(d)}\left(\Omega_{R}\right)$. On commence d'abord par établir le lemme suivant.

Lemme 4.16. Soient $R>0$ fixé et $u(t, x) \in G^{\omega,(d)}\left(\Omega_{R}\right)$ tel que $u(0, x) \equiv 0$ dans $\Omega$. Alors, pour tout $\rho>0, \eta>1$, pour tout ouvert $\Omega^{\prime}$ relativement compact dans $\Omega$ et tout $r>0$ tel que $\bar{D}_{4^{s^{\prime}} \eta r} \subset D_{R}$, on a $u \in \mathcal{C}_{\Phi_{\rho, r}}^{\omega, d}\left(\Omega_{r}^{\prime}\right)$.

Preuve. Soit $R>0$ fixé. Soient $\eta>1, \rho>0$ et $\Omega^{\prime}$ un ouvert relativement compact dans $\Omega . u(t, x)$ étant entière par rapport à $t$, alors d'aprés les inégalités de Cauchy pour tout $r>0$ tel que $\bar{D}_{4^{s^{\prime}} \eta r} \subset D_{R}$, il existe $c \geq 0$ tel que:

$$
\begin{aligned}
\forall \alpha \in \mathbb{N}^{n}, \forall(t, x) \in \bar{D}_{4^{s^{\prime}} \eta r} \times \Omega^{\prime}, \\
\qquad D_{x}^{\alpha} u(t, x) \ll c\left(\frac{1}{\eta \rho r}\right)^{|\alpha|}(|\alpha| !)^{d}\left(\frac{\eta \rho r}{\eta \rho r-\left(\rho / 4^{s^{\prime}}\right) \sigma}-1\right) .
\end{aligned}
$$

En raisonnant comme pour le lemme 4.11 on obtient pour tout $r>0$ tel que $\bar{D}_{4^{s^{\prime}} \eta r} \subset D_{R}$ et tout $(t, x) \in \Omega_{r}^{\prime} \subset \bar{D}_{4^{s^{\prime}} \eta r} \times \Omega^{\prime}$;

$$
\begin{aligned}
u(t, x) & \ll c \sum_{j=0}^{+\infty}(\xi / \eta \rho r)^{j}(j !)^{d-1}\left(1 / 4^{s^{\prime}} \eta r\right) \sigma \frac{\eta \rho r}{\eta \rho r-\left(\rho / 4^{s^{\prime}}\right) \sigma} \\
& \ll(c / r) \sigma\left(\sum_{j=0}^{+\infty}(\xi / \eta \rho r)^{j} \frac{\eta \rho r}{\eta \rho r-\left(\rho / 4^{s^{\prime}}\right) \sigma}\right)^{d} \\
& \ll c(\eta)(c / r) \Phi_{\rho, r}^{d}(t, x) .
\end{aligned}
$$


4.3.1. Preuve de la proposition 3.4. Soit $R>0$ fixé. D'aprés le lemme 4.15. l'équation (9) admet une solution $u \in G^{\omega,(d)}\left(\Omega_{R}\right)$. $\Omega$ étant la réunion de tous les ouverts relativement compacts de $\Omega$ alors, pour montrer que $u$ est unique dans $\Omega_{R}$ il suffit de montrer que $u$ est unique dans $D_{R} \times \Omega^{\prime}$ pour tout ouvert $\Omega^{\prime}$ relativement compact dans $\Omega$. Soient $u_{1}, u_{2}$ deux solutions de l'équation (9) dans $G^{\omega,(d)}\left(\Omega_{R}\right)$, alors $u_{1}$ et $u_{2}$ sont deux points fixes de l'application $\mathcal{F}$ dans $G^{\omega,(d)}\left(\Omega_{R}\right)$ et $u_{1}(0, x)=u_{2}(0, x) \equiv 0$ dans $\Omega$. Si $\Omega^{\prime}$ est un ouvert relativement compact de $\Omega$, alors d'aprés le lemme 4.16 pour tout $\rho>0, \eta>1$, pour tout $r>0$ tel que $\bar{D}_{4^{s^{\prime}} \eta r} \subset D_{R}$, on a $u_{1}, u_{2} \in \mathcal{C}_{\Phi \rho, r}^{\omega, d}\left(\Omega_{r}^{\prime}\right)$ et donc, d'aprés la proposition 4.13 il existe $\rho_{0}>0$ tel que pour tout $\rho \geq \rho_{0}, \mathcal{F}$ est une contraction stricte de la boule $B\left(0, a_{\rho}\right)$ de $\mathcal{C}_{\Phi}^{\omega, d}\left(\Omega_{r}^{\prime}\right)$. Ainsi, d'aprés la proposition 4.14 et par unicité du point fixe dans $\mathcal{C}_{\Phi_{\rho, r}}^{\omega, d}\left(\Omega_{r}^{\prime}\right)$, on déduit que $u_{1}=u_{2}$ dans $D_{r} \times \Omega^{\prime}$.

De plus, $u_{1}, u_{2} \in \mathcal{C}^{\omega, \infty}\left(\Omega_{R}\right)$, donc $u_{1}(., x)$ et $u_{2}(., x)$ sont holomorphes sur $D_{R}$ pour tout $x \in \Omega^{\prime}$. Sachant que $D_{r} \subset \bar{D}_{4^{s^{\prime}} \eta r} \subset D_{R}$ et que $D_{R}$ est connexe, alors par prolongement analytique on obtient $u_{1}(t, x)=u_{2}(t, x)$ dans $D_{R} \times \Omega^{\prime}$.

\section{REFERENCES}

[1] M.S. Baounendi, C. Goulaouic, Cauchy problems with characteristic initial hypersurface. Comm. on Pure and Appl. Math., 26 (1973), pp. 455-475. MR0338532 (49:3296)

[2] F. Derrab, A. Nabaji, P. Pongérard, C. Wagschal, Problème de Cauchy Fuchsien dans les espaces de Gevrey. J. Math. Sci. Univ. Tokyo., 11, No. 4, (2004), pp. 401-424. MR2110921 (2005i:35007)

[3] F. Derrab, A. Nabaji, Solutions holomorphes locale et globale pour un opérateur différentiel linéaire à plusieurs variables Fuchsiennes. Osaka J. Math., 42, No. 3, (2005), pp. 653-675. MR.2166727

[4] F. Derrab, Sur les équations BMG. Thèse de Doctorat d'État, Sidi-Bel-Abbès (2005).

[5] R. Gérard, H. Tahara, Singular nonlinear partial differential equations. Vieweg, Wiesdaden (1996). MR 1757086 (2001c:35056)

[6] M. Gevrey, Sur la nature analytique des solutions des équations aux dérivées partielles. Ann. Sci. École Norm. Sup., 35 (1918), pp. 129-190. MR1509208

[7] H. Komatsu, Linear hyperbolic equations with Gevrey coefficients. J. Math. Pures et Appl., 59 (1980), pp. 145-185. MR0581987 (82f:35114)

[8] P. D. Lax, Non linear hyperbolic equations. Comm. Pure Appl. Math, 6 (1953), pp. 231-258. MR.0056176 (15:36a)

[9] N. S. Madi, M. Yoshino, Uniqueness and solvability of nonlinear Fuchsian equations. Bull. Sci. Math., $2^{e}$ série., 114 (1990), pp. 41-60. MR1046700 (92b:35009)

[10] M. Miyake, Singular nonlinear partial differential equations of the first order in a complex domain. Séminaire Adjamagbo, Paris 9, Décember (1997).

[11] P. Pongérard, Sur une classe d'équations de Fuchs non-linéaires. J. Math. Sci. Univ. Tokyo., 7 (2000), pp. 423-448. MR.1792735 (2001j:35003)

[12] P. Pongérard, Problème de Cauchy caratéristique à solution entières. J. Math. Sci. Univ. Tokyo., 8 (2001), pp. 89-105. MR1818907 (2001m:35009)

[13] C. Wagschal, Le problème de Goursat non linéaire. J. Math. Pure et Appl., 58 (3), (1979), pp. 309-337. MR0544256 (82m:35024)

86, Avenue Lieutenant Khelladi, 22000 Sidi-Bel-Abbès, Algérie

E-mail address: nouveaucompte2003@yahoo.fr 